\title{
A Rare Case of End-Stage HF Salvaged by Rescue Cardiac Resyn- chronization Therapy (CRT)
}

\author{
Yaser Khalid, DO ${ }^{1^{*}}$, Neethi Dasu, DO ${ }^{1}$, Kirti Dasu, BA ${ }^{2}$, Paul Jang, DO ${ }^{1}$ and Vivek Sailam ${ }^{3}$ \\ ${ }^{1}$ Rowan School of Medicine, Jefferson Health System, USA \\ ${ }^{2}$ Department of Biology, Syracuse University, USA \\ ${ }^{3}$ Department of Cardiology, Virtua Lourdes, USA
}

*Corresponding author: Dr. Yaser Khalid, DO, Rowan School of Medicine, Jefferson Health System, One Medical Center Dr, UDP Suite 2300 Stratford, NJ 08084, USA, Tel: 6093503615

\begin{abstract}
Background: End-stage heart failure (HF) is defined as a lack of improvement of HF symptoms and frequent exacerbations despite maximal medical therapy. Current guidelines recommend against the use of Cardiac Resynchronization Therapy (CRT) as a rescue device in end-stage HF.

Case Report: We present a case of an unconventional approach using CRT to treat refractory end-stage HF.

Conclusions: CRT is a promising intervention for new-onset end-stage HF for patients who may be able to recover enough of their EF to restart goal-directed medical therapy (GDMT). This report highlights the promising clinical outcome of an unconventional CRT use in end-stage HF despite a level $C$ recommendation in current guidelines. Further studies are required to validate these findings and to establish the causes of these observations.
\end{abstract}

\section{Keywords}

CRT, Cardiogenic shock, Inotropes, HF

\section{Abbreviations}

CRT: Cardiac Resynchronization Therapy; LVAD: Left Ventricular Assist Device; CABG: Coronary Artery Bypass Graft; BNP: Brain Natriuretic Peptide; CXR: Chest X-ray; EKG: Electrocardiogram; LBBB: Left Bundle Branch Block; TTE: Transthoracic Echocardiogram; NYHA: New York Heart Association; HF: Heart Failure

\section{Introduction}

End-stage heart failure (HF) is defined as a lack of improvement of HF symptoms and frequent exacerbations despite maximal medical therapy. Current guidelines recommend against the use of Cardiac Resynchronization Therapy (CRT) as a rescue device in end-stage HF. We present a case of an unconventional approach using CRT to treat refractory end-stage HF. CRT is a promising intervention for new-onset end-stage HF for patients who may be able to recover enough of their EF to restart GDMT. This report highlights the promising clinical outcome of an unconventional CRT use in end-stage HF despite a level $\mathrm{C}$ recommendation in current guidelines.

\section{Case Report}

A 76-year-old male presented with two days of worsening shortness of breath, hallucinations, and confusion. His home medications were rosuvastatin, icosapent ethyl, fenofibrate, aspirin, clopidogrel, sacubitril/ valsartan, carvedilol, isosorbide mononitrate, and torsemide. On examination, his blood pressure was $72 / 50$ $\mathrm{mmHg}$, pulse rate was 80 beats/min and regular, respiratory rate was 16 breaths/min, oxygen saturation was $99 \%$ on $2 \mathrm{~L}$ oxygen nasal cannula, and his temperature was $102.3^{\circ} \mathrm{F}$. He was confused, had mild respiratory distress, along with a S3, jugular venous distention, and a holosystolic murmur at the left $5^{\text {th }}$ intercostal space. He also presented with $2+$ lower extremity edema and $1+$ pretibial edema. He had a medical history of nonrevascularizable severe multivessel coronary artery disease status-post coronary artery bypass graft (CABG), class IV systolic and diastolic congestive $\mathrm{HF}$, severe peripheral vascular disease, hypertension, diabetes mellitus, dyslipidemia, and obstructive sleep apnea.

Citation: Khalid Y, Dasu N, Dasu K, Jang P, Sailam V, et al. (2020) A Rare Case of End-Stage HF Salvaged by Rescue Cardiac Resynchronization Therapy (CRT). Int J Clin Cardiol 7:181. doi.org/10.23937/23782951/1410181 


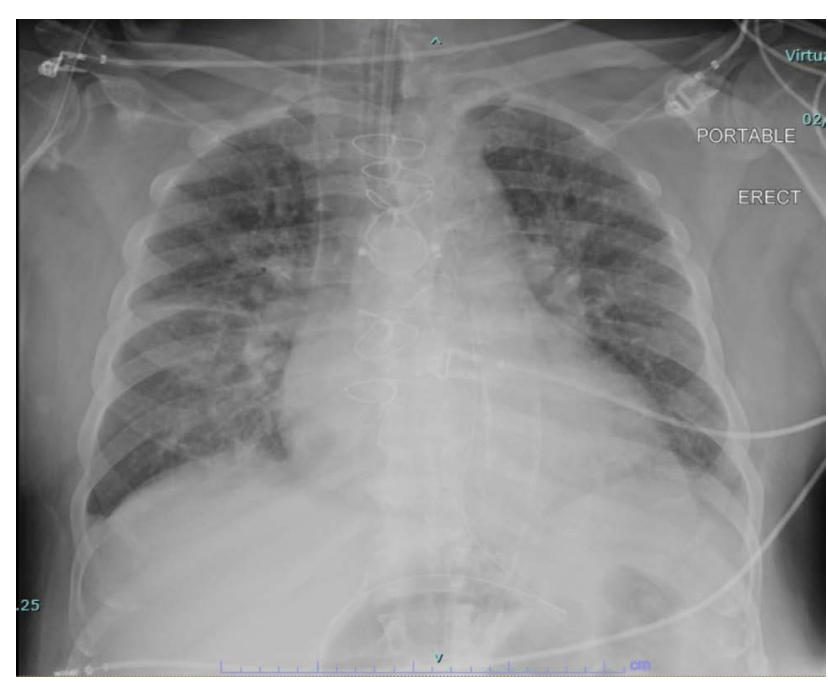

Figure 1: CXR on initial presentation showing bilateral diffuse pulmonary edema.
Laboratory results were the following: Brain natriuretic peptide (BNP) greater than $5000 \mathrm{ng} / \mathrm{L}$ and a creatinine of $2.8 \mathrm{mg} / \mathrm{dL}$ (increased from $1.7 \mathrm{mg} / \mathrm{dL}$ from 2 months prior). Chest x-ray (CXR) demonstrated cephalization, pulmonary vascular congestion, and diffuse bilateral edema (Figure 1). Electrocardiogram (EKG) showed normal sinus rhythm, left bundle branch block (LBBB) with a QRS of $124 \mathrm{~ms}$, and nonspecific ST-T wave changes (Figure 2). A transthoracic echocardiogram (TTE) showed an EF of 20-25\% (Figure 3 and Figure 4) with globally reduced wall motion function.

The patient was restarted on his home medications and given intravenous (IV) lasix, which improved the patient's mental status and generalized edema. However, his creatinine increased, and nephrology recommended holding his sacubitril/valsartan and carvedilol due to persistent hypotension. He was then noted to

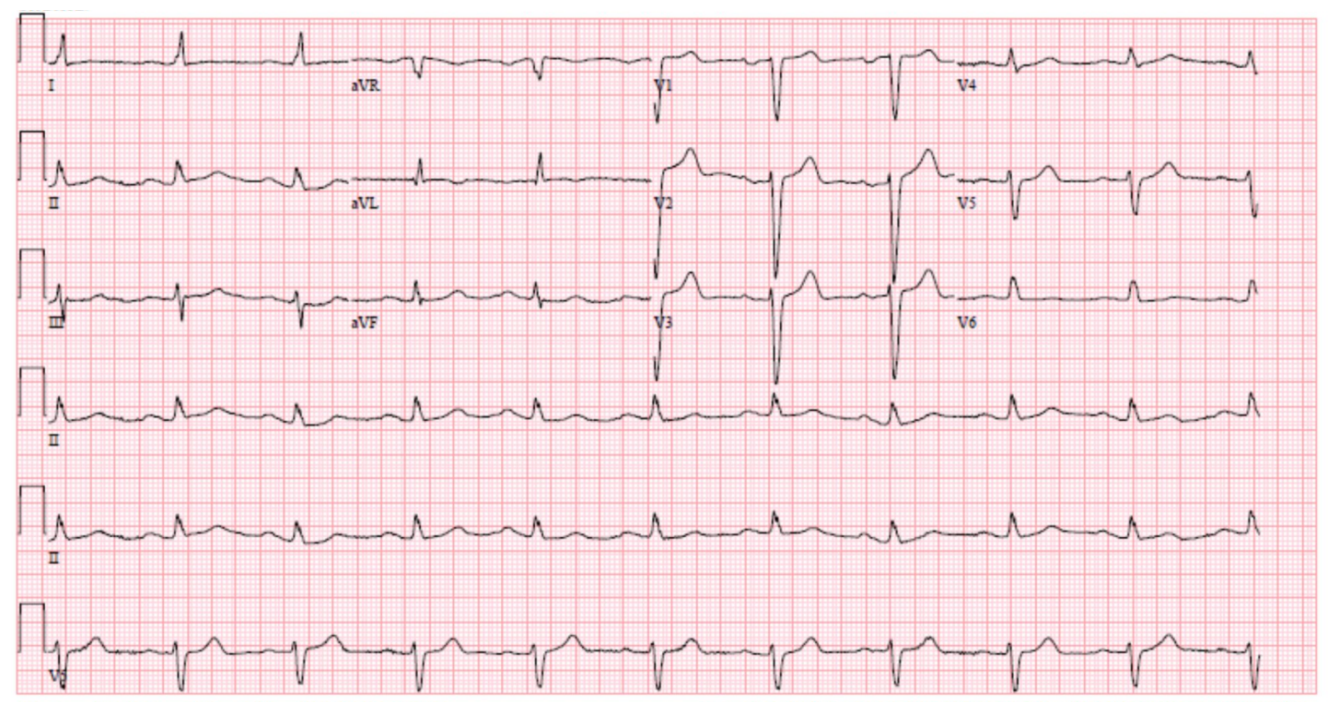

Figure 2: EKG with NSR, LBBB with QRS 124 ms.

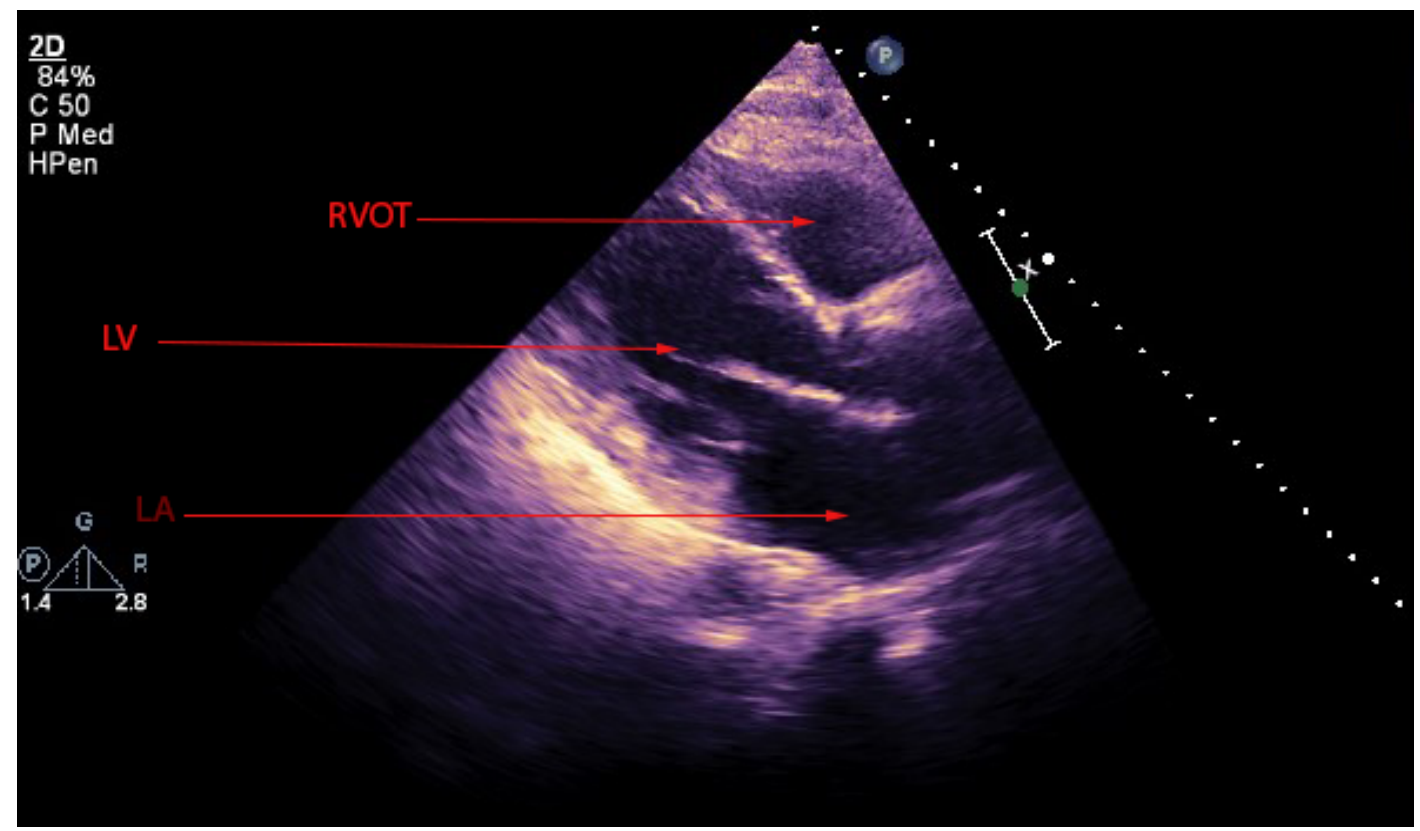

Figure 3: TTE parasternal long echocardiogram view. 


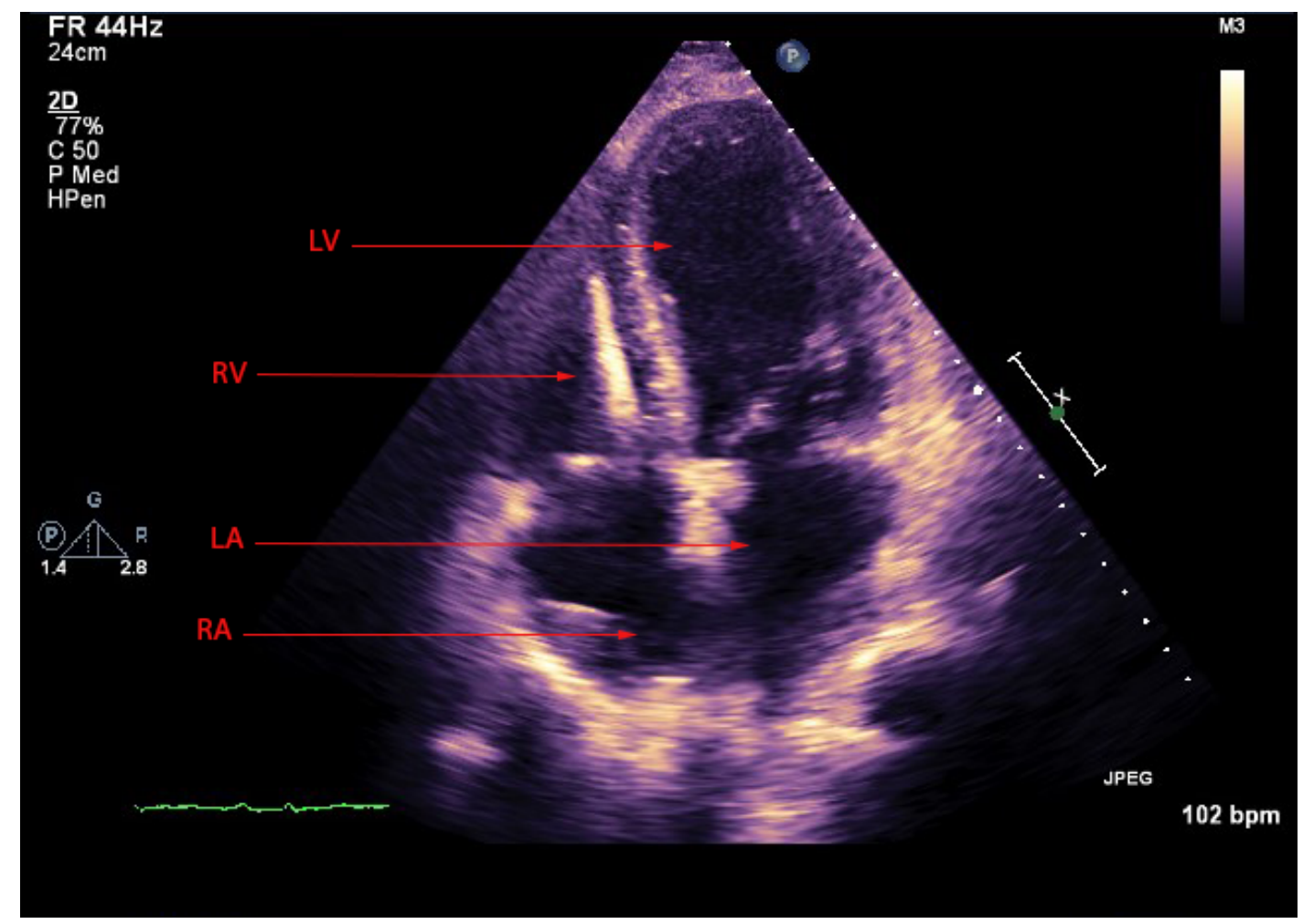

Figure 4: TTE apical four chamber echocardiogram view.

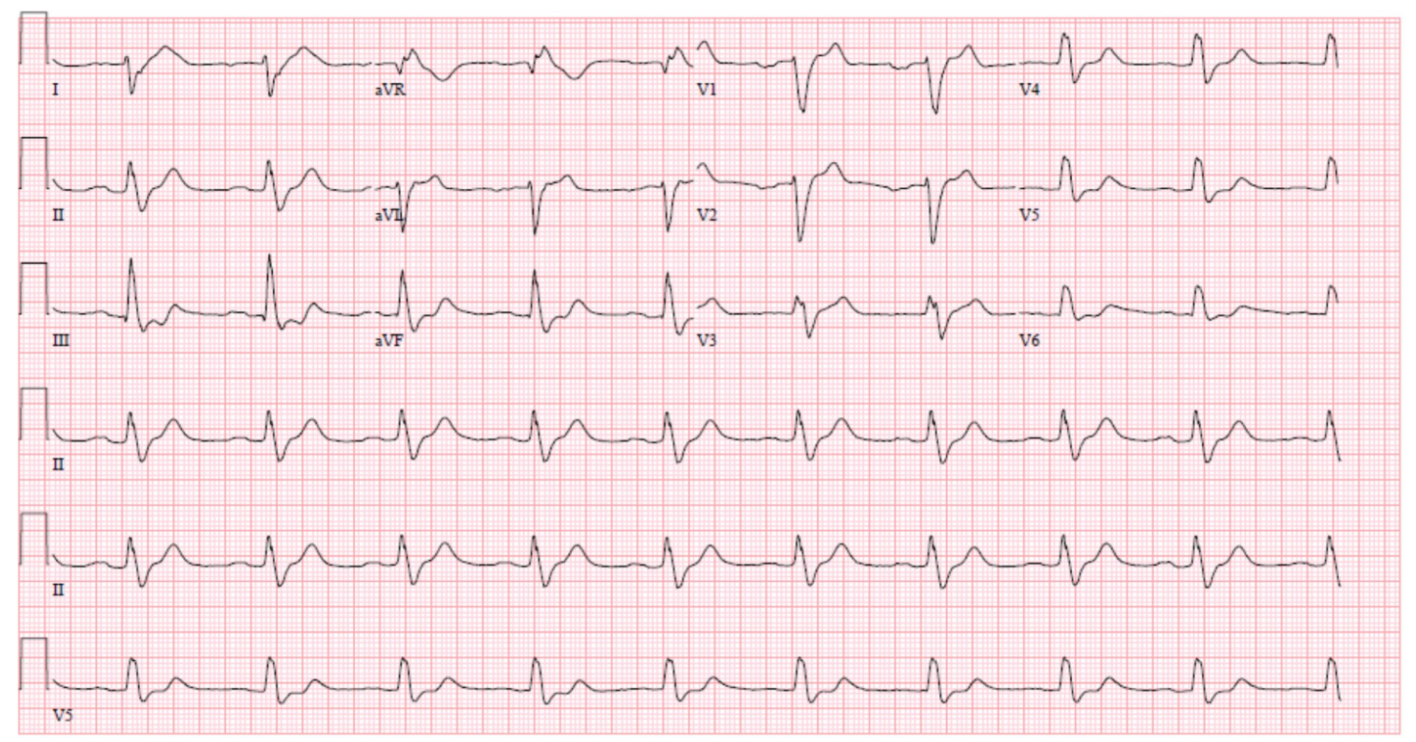

Figure 5: EKG with NSR, LBBB with QRS 213 ms.

have worsening dyspnea on exertion, episodes of hallucinations, and confusion. The patient was intubated and upgraded to the intensive care unit due to an increased work of breathing with hypoxia. He was diagnosed with end-stage HF and cardiogenic shock based on clinical and physical exam findings of cool extremities, altered mental status, and decreasing heart rate. He was started on dobutamine and norepinephrine infusions with improvement in all of his clinical symptoms.

After three days, the patient was extubated, but he could not be weaned from the dobutamine infusion. Palliative care recommended home hospice with a palli- ative dobutamine infusion. A repeat EKG showed worsening LBBB now with a QRS of $213 \mathrm{~ms}$ (Figure 5). Electrophysiology recommended cardiac resynchronization therapy (CRT) with a defibrillator because the patient met EKG criteria for the device with a wide QRS of 230 $\mathrm{ms}$ and a LBBB as well as $\mathrm{EF}<35 \%$ requiring an implantable cardiac defibrillator (ICD). Prior to CRT placement, hemodynamic device optimization by stroke work with quadripolar leads was performed, which has recently shown an additional one-third pump function improvement.

After CRT, the patient was hemodynamically stable 


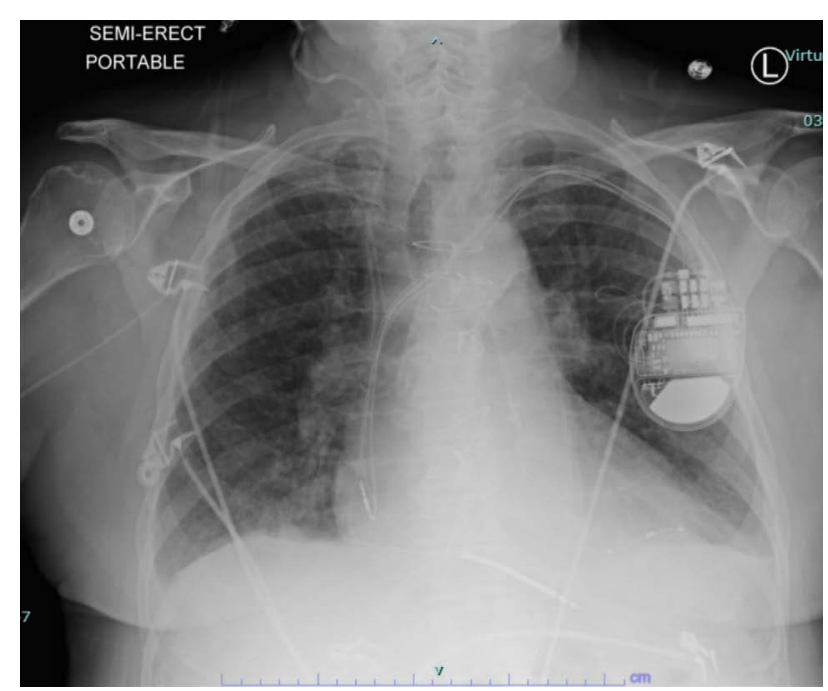

Figure 6: CXR with resolved pulmonary edema. with cessation of the dobutamine infusion. His kidney function continued to improve with a creatinine of 1.22 $\mathrm{mg} / \mathrm{dL}$ on the day of discharge - the lowest it was noted in years. A repeat EKG showed biventricular pacing and LBBB with a QRS of $133 \mathrm{~ms}$ (Figure 6). A repeat CXR showed resolution of the pulmonary edema (Figure 7). After discharge, he followed up with his HF clinic, and a repeat TTE showed an improved EF of $30-35 \%$ (Figure 8 , Figure 9 and Figure 10). After discharge, a repeat TTE showed an improved EF of 30-35\% (Figure 8, Figure 9 and Figure 10) and was restarted on maximum GDMT.

\section{Discussion}

The benefits of CRT have been firmly established in HF patients who remain in New York Heart Association (NYHA) functional classes II and III despite optimal medical therapy with a wide QRS complex greater than QRS

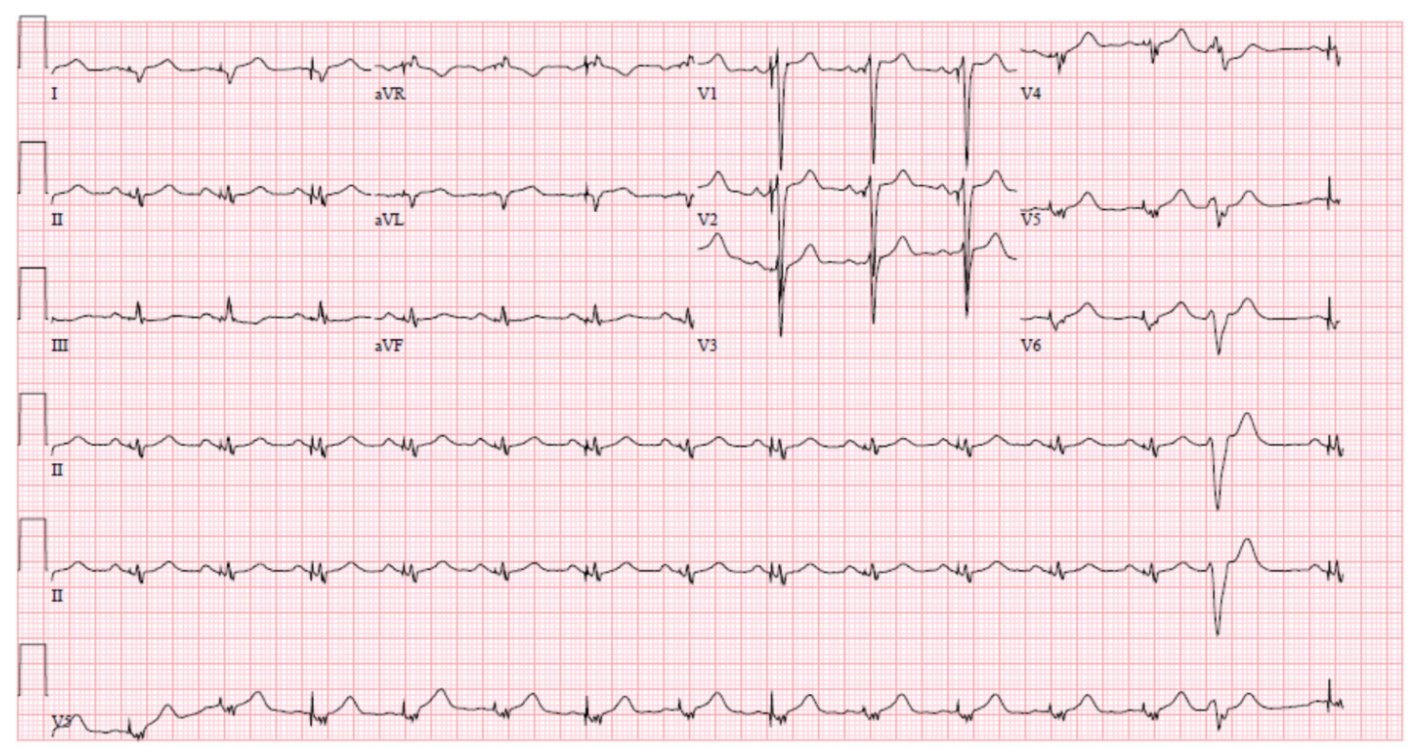

Figure 7: EKG with NSR, LBBB with QRS 133 ms.

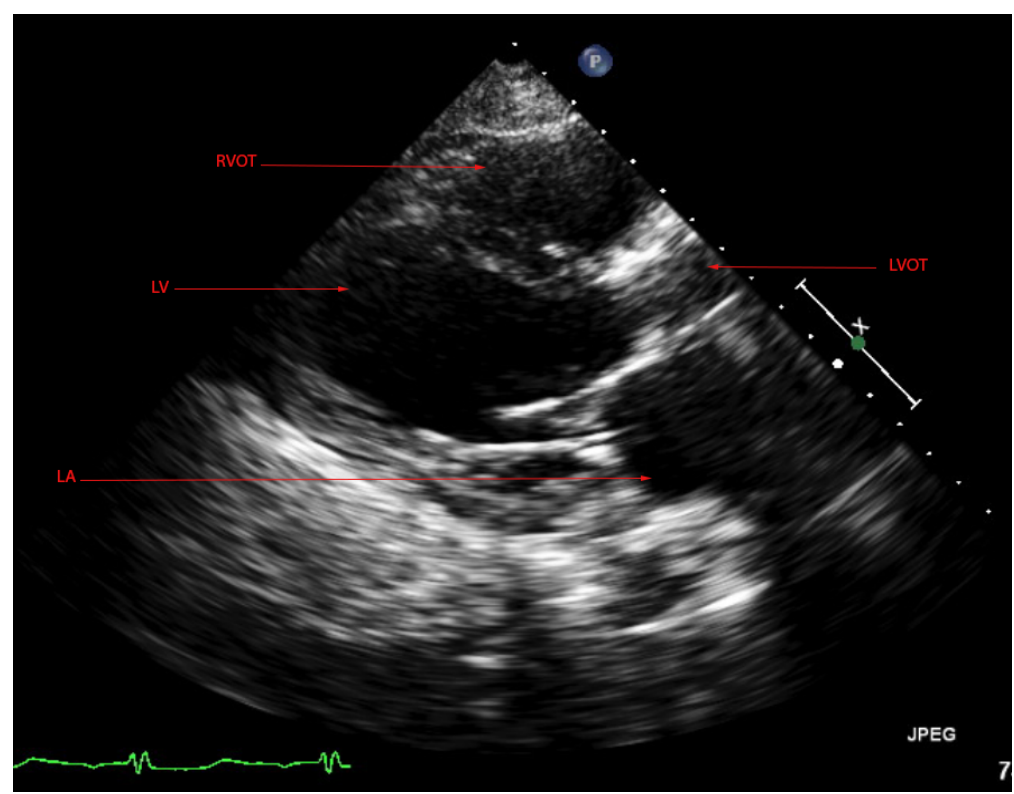

Figure 8: TTE parasternal long echocardiogram view after CRT. 


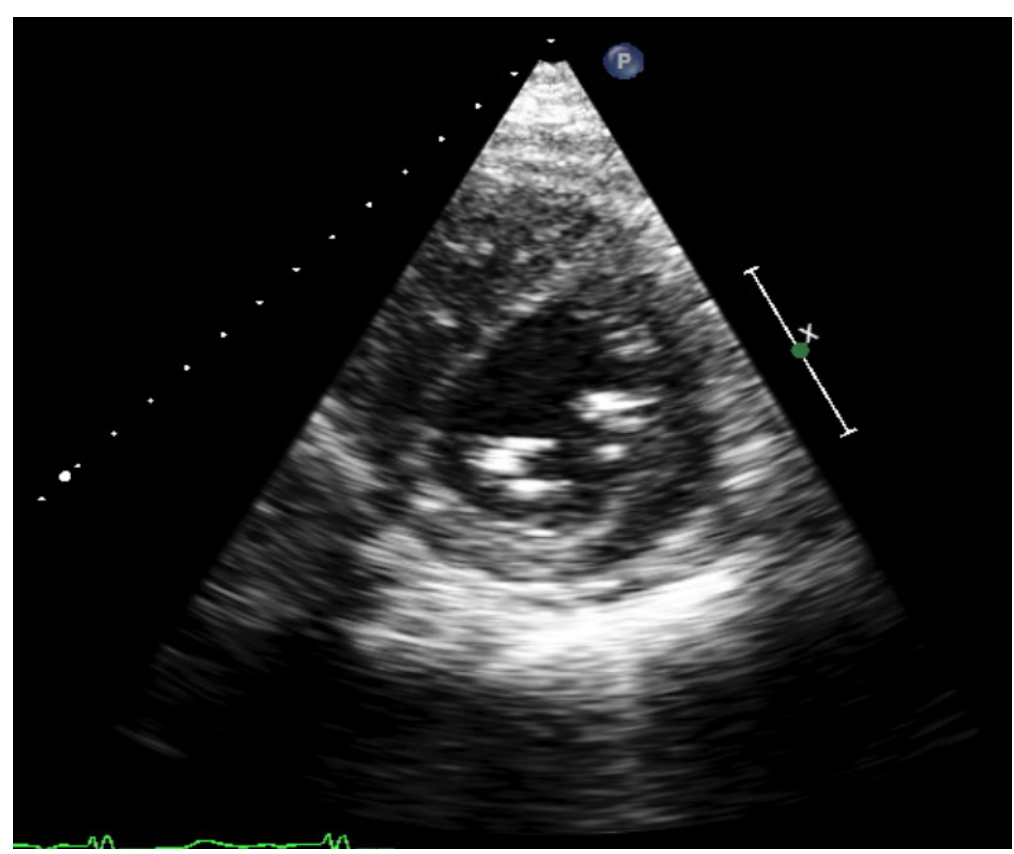

Figure 9: TTE parasternal short echocardiogram view after CRT.

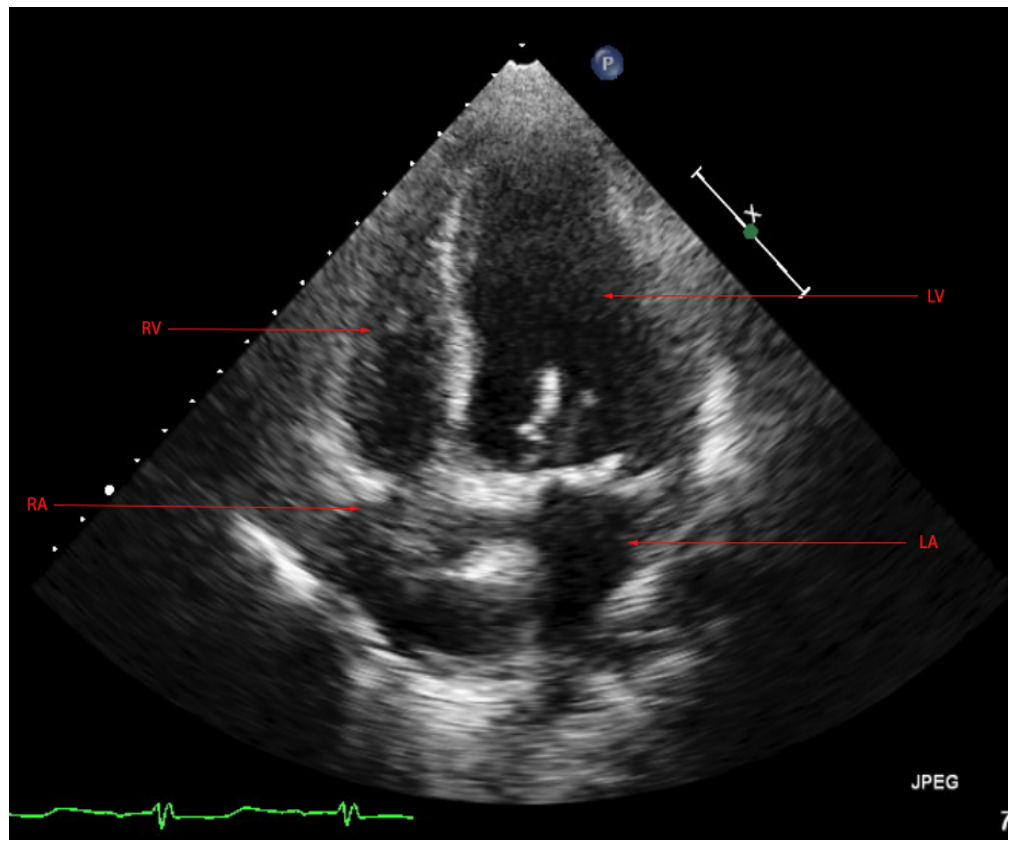

Figure 10: TTE apical four chamber echocardiogram view after CRT.

of $150 \mathrm{~ms}$ and a reduced left ventricular ejection fraction $\leq 30 \%$ to $35 \%$ [1]. Currently, there is a strong consensus in international guidelines on CRT implantation indications. The majority of cardiac societies' guidelines recommend that patients with a LBBB and a QRS duration $\geq 150 \mathrm{~ms}$ should be offered a CRT device provided they are in NYHA functional class II and ambulatory [2]. However, for certain patients on vasopressors and with a life expectancy of less than one year, there are no clear guidelines [2].

There are only a few contraindications to CRT. Inotrope-dependent patients have a perpetually high risk of death emphasizing the need for cardiac transplant or left-ventricle assist device (LVAD) support regardless of
CRT implantation and use [3]. The benefits of CRT in this population appear to be limited to a level $\mathrm{C}$ recommendation according to the American College of Cardiology Foundation/American Heart Association guidelines published in 2013 [4]. Therefore, CRT is generally avoided in this patient population unless a strong indication exists.

The decision to insert the CRT despite meeting the definition of end-stage HF was based on multiple factors. The patient was previously relatively functional, had a good history of compliance to maximum GDMT, and the guideline-based treatment recommendations with his declined HF classification was reduced to palliative at-home care. In early studies assessing the hemodynamic benefits of biventricular pacing, immediate 
improvement in cardiac index and pulmonary capillary wedge pressure was demonstrated after the insertion of a CRT device $[5,6]$. With this confluence of factors and a previous consideration for CRT adding weight to the decision, the benefit assessment began to outweigh the risks. Our patient benefited from CRT placement despite his limited life expectancy and tenuous clinical condition. His EF improved from $20-25 \%$ to $30-35 \%$ and we were able to restart his GDMT. The mechanism of acute pump function improvement was synchrony of the septal wall with the other walls of the heart for improved contraction due to our patient's underlying LBBB.

This case demonstrates the value of CRT as a rescue device in patients acutely decompensating into endstage HF, improving cardiac function enough to stabilize the patient to a degree in which he no longer met criteria for end-stage HF. This was an unexpected, but welcome outcome. Investigating the utility of CRT as a rescue device promises potential for improving outcomes especially in the patient population described. Additionally, based on this evidence, guidelines ruling out the viability of CRT need to be reconsidered. For patients whose options are severely limited by disease state or declining clinical presentation, CRT offers a rare chance to return to a manageable hemodynamic state and eventual transition back to GDMT.

\section{Conclusions}

Our case illustrates that benefits from CRT may exist outside of the guidelines selection criteria in individual cases. Further studies are required to validate these findings and to establish the causes of these observa- tions.

\section{Funding Information}

No funding or financial support has been allocated for this article.

\section{Disclosures}

All authors have no relevant disclosures with regards to this article.

\section{References}

1. Roger VL, Weston SA, Redfield MM, Hellermann-Homan JP, Killian J, et al. (2004) Trends in heart failure incidence and survival in a community-based population. JAMA 292: 344-350.

2. Normand C, Linde C, Singh J, Dickstein K (2018) Indications for cardiac resynchronization therapy: A Comparison of the major international guidelines. JACC Heart Fail 6: 308-316.

3. Vahdatpour C, Collins D, Goldberg S (2019) Cardiogenic shock. J Am Heart Assoc 8: e011991.

4. Yancy CW, Jessup M, Bozkurt B, Butler J, Casey Jr DE, et al. (2013) 2013 ACCF/AHA guideline for the management of heart failure: A report of the American College of Cardiology Foundation/American Heart Association task force on practice guidelines. J Am Coll Cardiol 62: e147-e239.

5. Foster AH, Gold MR, McLaughlin JS (1995) Acute hemodynamic effects of atrio-biventricular pacing in humans. The Annals of Thoracic Surgery 59: 294-300.

6. Leclercq C, Cazeau S, Le Breton H, Ritter P, Mabo P, et al. (1998) Acute hemodynamic effects of biventricular DDD pacing in patients with end-stage heart failure. Journal of the American College of Cardiology 32: 1825-1831. 